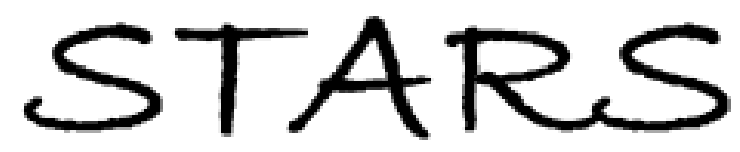

University of Central Florida

STARS

$1-1-2013$

\title{
Reconfigurable fabrication of scattering-free polymer network liquid crystal prism/grating/lens
}

Jie Sun

University of Central Florida

Su Xu

University of Central Florida

Hongwen Ren

Shin-Tson Wu

University of Central Florida

Find similar works at: https://stars.library.ucf.edu/facultybib2010

University of Central Florida Libraries http://library.ucf.edu

This Article is brought to you for free and open access by the Faculty Bibliography at STARS. It has been accepted for inclusion in Faculty Bibliography 2010 s by an authorized administrator of STARS. For more information, please contact STARS@ucf.edu.

\section{Recommended Citation}

Sun, Jie; Xu, Su; Ren, Hongwen; and Wu, Shin-Tson, "Reconfigurable fabrication of scattering-free polymer network liquid crystal prism/grating/lens" (2013). Faculty Bibliography 2010s. 4726.

https://stars.library.ucf.edu/facultybib2010/4726

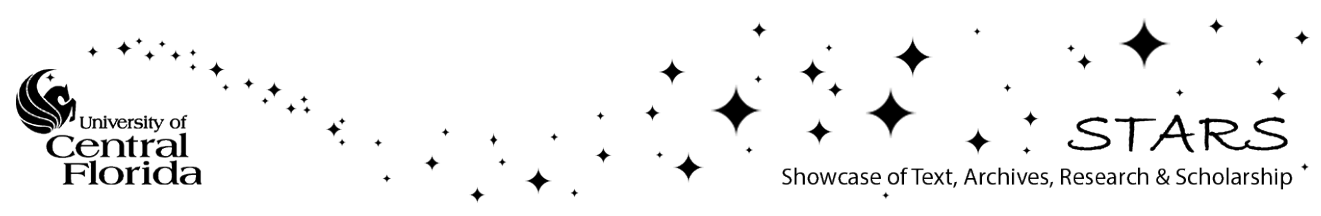




\section{Reconfigurable fabrication of scattering- free polymer network liquid crystal prism/ grating/lens}

Cite as: Appl. Phys. Lett. 102, 161106 (2013); https://doi.org/10.1063/1.4802919

Submitted: 03 March 2013. Accepted: 10 April 2013 . Published Online: 23 April 2013

Jie Sun, Su Xu, Hongwen Ren, and Shin-Tson Wu
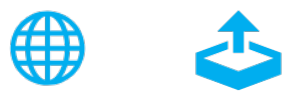

View Online

Export Citation

\section{ARTICLES YOU MAY BE INTERESTED IN}

Fast-response and scattering-free polymer network liquid crystals for infrared light modulators

Applied Physics Letters 84, 1233 (2004); https://doi.org/10.1063/1.1649816

A low voltage submillisecond-response polymer network liquid crystal spatial light modulator Applied Physics Letters 104, 023305 (2014); https://doi.org/10.1063/1.4862474

Polarizer-free and fast response microlens arrays using polymer-stabilized blue phase liquid crystals

Applied Physics Letters 96, 113505 (2010); https://doi.org/10.1063/1.3360860

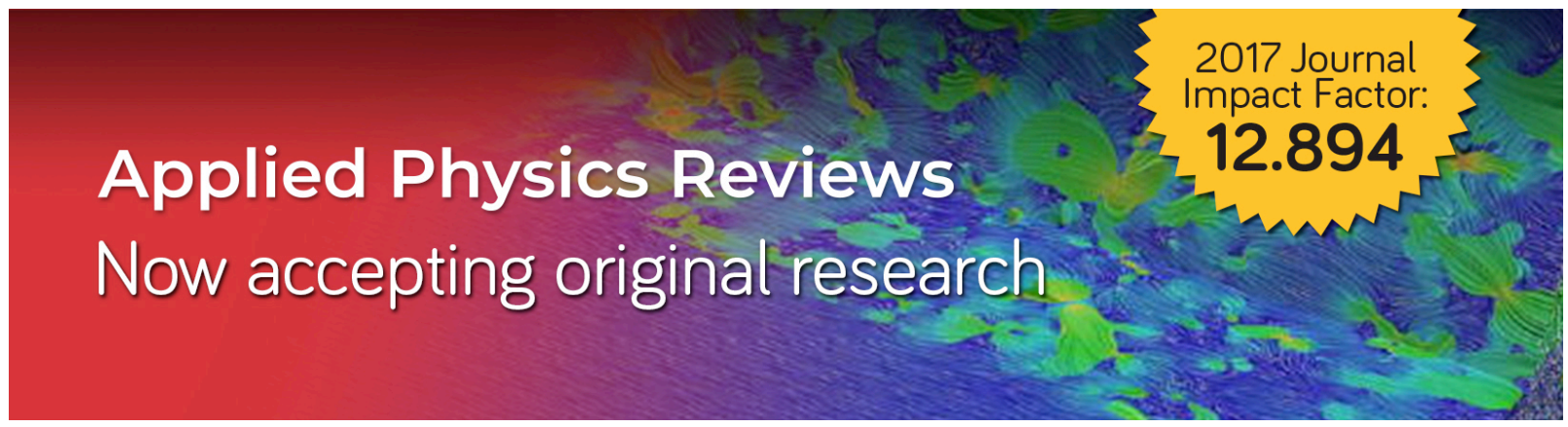




\title{
Reconfigurable fabrication of scattering-free polymer network liquid crystal prism/grating/lens
}

\author{
Jie Sun, ${ }^{1}$ Su Xu, ${ }^{1}$ Hongwen Ren, ${ }^{2}$ and Shin-Tson $\mathrm{Wu}^{1, \mathrm{a})}$ \\ ${ }^{1}$ CREOL, The College of Optics and Photonics, University of Central Florida, Orlando, Florida 32816, USA \\ ${ }^{2}$ Department of Polymer Nano Science and Technology, Chonbuk National University, Jeonju, Jeonbuk, \\ South Korea
}

(Received 3 March 2013; accepted 10 April 2013; published online 23 April 2013)

\begin{abstract}
We report a simple reconfigurable printing method for fabricating submillisecond-response and scattering-free polymer network liquid crystal photonic devices, such as prism, grating, and lens. To suppress light scattering in the visible region, we reduce the domain sizes by controlling polymer concentration, selecting a high viscosity liquid crystal (LC) host, and performing UV curing at a low temperature. To demonstrate the configurability, we printed a LC micro-prism array with $\sim 300-\mu \mathrm{m}$ feature size and a circular lens with $1.3-\mathrm{mm}$ radius without any pre-patterned templates. This reconfigurable printing technique enables fast design iterations and should have widespread applications for fabricating display and photonic devices. ( 2013 AIP Publishing LLC [http://dx.doi.org/10.1063/1.4802919]
\end{abstract}

Adaptive liquid crystal (LC) grating, ${ }^{1-5}$ lens, ${ }^{6-10}$ and prism $^{11,12}$ are intriguing devices because their electro-optic properties can be tuned by an external voltage. To fabricate these photonic devices, a pre-patterned template, such as patterned electrodes or photomask, is commonly used, which requires expensive instruments and time consuming procedures. A typical photolithographic procedure to fabricate patterned electrodes includes applying photoresist, exposure, etching, and photoresist removal. Recently, a microlithography system based on reflective digital micromirror device for generating high resolution arbitrary photoalignment patterns in LC cells has been demonstrated. ${ }^{13}$ This approach is reconfigurable and particularly attractive for making switchable LC grating, except that the optical system is rather complicated.

In addition to the abovementioned sophisticated fabrication procedure, the device performance, especially response time, is another serious concern. The response time of a nematic LC is mainly governed by the cell gap, visco-elastic constant of the employed LC material, and voltage. ${ }^{14}$ For a LC lens with 30- $\mu \mathrm{m}$ cell gap, the response time is in the order of $1 \mathrm{~s} .{ }^{15}$ To shorten response time, thin cell with a high birefringence LC material, ${ }^{16}$ overdrive and undershoot voltage method, ${ }^{17,18}$ and polymer network liquid crystal (PNLC) ${ }^{19-25}$ have been investigated, just to name a few. For a PNLC containing 6-7 wt. \% polymer, the response time can be reduced to submillisecond while maintaining a $2 \pi$ phase change. ${ }^{23}$ However, there are two tradeoffs: (1) The operation voltage is increased dramatically due to strong polymer network, and (2) the light scattering remains noticeable due to submicron domain sizes formed by the LC molecules. Recently, submillisecond-response and scattering-free PNLCs have been demonstrated at $\lambda=1064 \mathrm{~nm}$ by reducing the domain sizes. However, light scattering in the visible region, say $\lambda=550 \mathrm{~nm}$, still exceeds $20 \% .^{23}$ To suppress light scattering, low density polymer-stabilized LC with polymer localized at the substrate surface has been developed. ${ }^{26,27}$ Since no

${ }^{a)}$ Electronic mail: swu@ucf.edu polymer network exists in the bulk LC, the operating voltage is low, and light scattering is negligible, but the response time is also relatively slow. There is an urgent need to overcome the above-mentioned problems, i.e., reconfigurable and simple fabrication process, while keeping submillisecond response time and negligible scattering loss.

In this letter, we report a scattering-free PNLC in the visible region and then present a reconfigurable one-step printing technique for fabricating LC micro-prism/lens/ grating. To demonstrate the feasibility and versatility of this technique, we printed a LC micro-prism array/grating with periodicity of $\sim 300 \mu \mathrm{m}$ and a circular LC lens with radius $\sim 1.3 \mathrm{~mm}$ as examples. The processing time for each device is about $10 \mathrm{~min}$. The functionality of these devices is also evaluated. Compared to other methods, our method shows advantages of easy configurability, low cost, and fast response time. This printing technique enables fast design iterations so that it could have widespread applications for fabricating display and photonics devices.

First, let us discuss our approach for obtaining scattering-free PNLC in the visible region. Increasing polymer concentration is known to be an effective way to shrink the PNLC domain sizes. ${ }^{19}$ However, high polymer concentration dramatically increases the device operating voltage. Recently, our group demonstrated a submillisecond-response and scattering-free $2 \pi$ phase modulator at $\lambda=1.06 \mu \mathrm{m}$ with only $6 \mathrm{wt}$. $\%$ polymer concentration. ${ }^{23} \mathrm{We}$ found that high viscosity LC host and low temperature UV curing play key roles for reducing the LC domain sizes and therefore suppressing light scattering. In order to extend the scatteringfree behavior to the visible region, we increased the polymer ratio from $6 \%$ to $10 \%$ to further reduce the domain size. To demonstrate the effectiveness of our approach, we prepared two PNLC precursors containing two different LC hosts (89.5\%), 10\% monomer (Merck RM257), and 0.5\% photoinitiator (GENOCURE BAPO). The LC host for PNLC-1 is HTG135200 (HCCH, China) with visco-elastic coefficient $\gamma_{1} / \mathrm{K}_{11}$ of $119.6 \mathrm{~ms} / \mu \mathrm{m}^{2}$ at $22^{\circ} \mathrm{C}$, birefringence $\Delta \mathrm{n}=0.21$ at 
$\lambda=633 \mathrm{~nm}$, and $\Delta \varepsilon=89$ at $1 \mathrm{kHz}$. The LC host for PNLC-2 is MLC14200 (Merck) with $\gamma_{1} / \mathrm{K}_{11}=27.3 \mathrm{~ms} / \mu \mathrm{m}^{2}$ at $22^{\circ} \mathrm{C}$, $\Delta \mathrm{n}=0.16$ at $\lambda=633 \mathrm{~nm}$ and $\Delta \varepsilon=25$. Commercial homogeneous LC cells with cell gap $d=5 \mu \mathrm{m}$ controlled by microbeads were employed. A UV lamp with $\lambda \sim 385 \mathrm{~nm}$ was employed for illumination. The curing temperature was controlled at $11^{\circ} \mathrm{C}$ for both PNLC cells.

Figure 1(a) shows the measured transmission spectra (unpolarized light) of PNLC-1 and PNLC-2 with a biased voltage $\left(\mathrm{V}_{\max }\right)$ leading to maximum scattering. The sample holder was set at $20 \mathrm{~cm}$ in front of the detector whose aperture is around $0.9 \mathrm{~cm}$. This corresponds to a collection angle of $\sim 3^{\circ}$. Such a small collection angle is to ensure that only transmitted light is collected by the detector. If the device has strong scattering, then the measured transmittance would be significantly reduced. The spectrum of a control nematic LC cell (HTG135200) is included in Fig. 1(a) for comparison. The small transmission oscillation is due to the FabryPerot effect of the ITO/LC interfaces. From Fig. 1(a), we can see that the scattering loss of PNLC-1 in the power-on state is negligible at $\lambda=633 \mathrm{~nm}$ (and $\sim 3 \%$ at $\lambda=480 \mathrm{~nm}$ ) in comparison with the nematic LC host. In contrast, the scattering loss of PNLC-2 in the visible region is much stronger (between $8 \%$ and $15 \%$ ). According to our previous study, the scattering loss of PNLCs generally follows the RayleighGans-Debye model. ${ }^{23}$ Without considering the reflection loss from interfaces, the transmittance of PNLC for a randomly polarized light at $\mathrm{V}_{\max }$ can be written as ${ }^{23}$

$$
T=\frac{1}{2} \exp \left(-C_{e} \frac{\Delta n^{2}}{\lambda_{0}^{2}} d\right)+\frac{1}{2} \exp \left(-C_{o} \frac{\Delta n^{2}}{\lambda_{0}^{2}} d\right),
$$

where $\mathrm{C}_{\mathrm{e}}$ and $\mathrm{C}_{\mathrm{o}}$ represent the domain size parameters for the extraordinary ray (e-ray) and ordinary ray (o-ray), respectively; $\lambda_{0}$ is the wavelength in vacuum. Therefore, small $\Delta \mathrm{n}$ and small domain sizes are helpful to reduce scattering loss. Considering MLC14200 used in PNLC-2 has a lower birefringence than HTG135200, we find that PNLC-1 must have much smaller domain sizes than PNLC-2 in order to explain its negligible scattering loss.

To further confirm these scattering characterization, we used a polarized green laser $(\lambda=514 \mathrm{~nm})$ to probe these two PNLCs at a power-on state. The polarization direction is
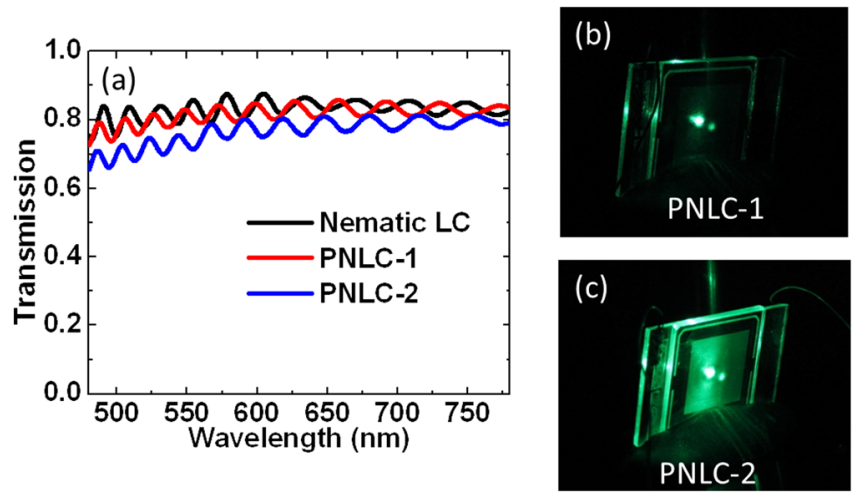

FIG. 1. (a) Transmission spectra of PNLC-1 (red line) and PNLC-2 (blue line) with $\mathrm{V}_{\max }$ applied. Transmission spectrum for a comparable nematic LC cell is shown for comparison (black line). (b), (c) Optical images of PNLC-1 and PNLC-2 with a green laser. parallel to the rubbing direction of the LC cells. Figures 1(b) and 1(c) show the captured optical images for PNLC-1 and PNLC-2, respectively. PNLC-1 is almost transparent to the laser beam while PNLC-2 heavily scatters light. This scattering-free PNLC enables us to develop useful photonic devices in the visible region.

Before discussing our printing technique for PNLC photonic devices, let us first describe the responsible physical mechanism of this scattering-free device. For a homogeneous aligned LC cell, its effective extraordinary refractive index $n_{\text {e-eff }}$ (light polarization parallel to the LC molecular alignment direction) is tunable by varying the applied voltage. If this nematic LC contains UV curable monomers, its on-state $n_{e-\text { eff }}$ would be "frozen" (polymer stabilized) by UV exposure, which can be approximately expressed as ${ }^{28}$

$$
n_{e-e f f}=\frac{n_{o} n_{e}}{\sqrt{n_{o}^{2} \sin ^{2} \theta+n_{e}^{2} \cos ^{2} \theta}},
$$

where $n_{o}$ and $n_{e}$ are the ordinary and extraordinary refractive index of the PNLC precursor, respectively, and $\theta$ is the effective tilt angle. After UV curing, the obtained PNLC cell is still electrically switchable but with a much faster response time at the tradeoff of increased operating voltage. Based on this mechanism, we are able to create any desired $n_{e}$ profile (within the refractive index range of the host LC) on the PNLC cell. Our group previously reported some initial attempts to fabricate PNLCs with gradient refractive index according to this principle. ${ }^{24}$ Here, we focus on the scattering-free photonic devices.

Next, we discuss the reconfigurable one-step printing technique for fabricating PNLC photonic devices. The experimental setup and fabrication procedures are schematically depicted in Fig. 2(a). A photomask, either a rectangular mask (Fig. 2(b)) (for prism, cylindrical lens, or grating) or an iris diaphragm (for circular lens), is connected to a motion controller (Newport ESP 300) and placed right above the LC cell. A computer with LabviEw software is used to control the timing and amplitude of the applied voltages. A UV lamp is used for stabilizing the polymer network. Figures 2(c) and 2(d) illustrate the fabrication procedures. At time $t_{1}$, a voltage $\mathrm{V}_{1}$ is applied to the cell and the LC molecules in the exposed region are stabilized at this state. At time $t_{2}$, a voltage $\mathrm{V}_{2}\left(\mathrm{~V}_{2}<\mathrm{V}_{1}\right)$ is applied. As the photomask keeps moving, LC molecules at the newly exposed stripe/ring are stabilized with a smaller tilt angle.

We use two examples to illustrate the configurability of our fabrication setup. The first example is to fabricate a onedimensional (1D) prism array. In order to "print" a prism array with known phase profile, we first obtained the electric field dependent $n_{\text {e-eff }}$ of the LC precursor through the electrooptical properties measurement (Fig. 3(a)). As the rectangular photomask is moving at a constant velocity $(0.01 \mathrm{~mm} / \mathrm{s}$ here $)$, we are able to convert the intended phase profile (Fig. 3(b)) into a time-dependent voltage chart (Fig. 3(c)). We wrote a MATLAB program which can do this conversion conveniently. A $15-\mu \mathrm{m}$ homogeneous LC cell was used here and below. The entire printing process of this prism took about $10 \mathrm{~min}$.

Figure 3(d) shows a polarized microscope photo (transmissive mode) of this prism array. Sample cell was sandwiched 
(a)

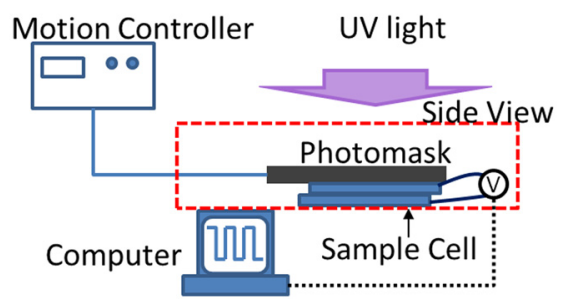

(b)

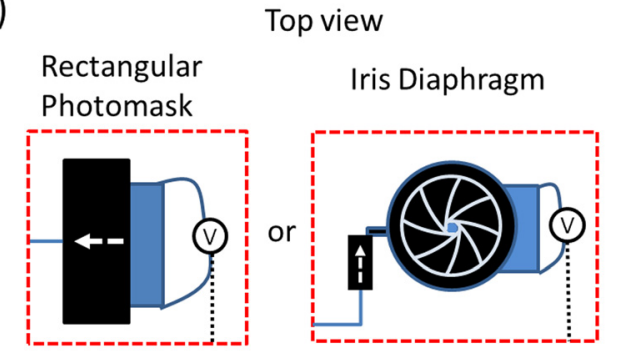

(c)
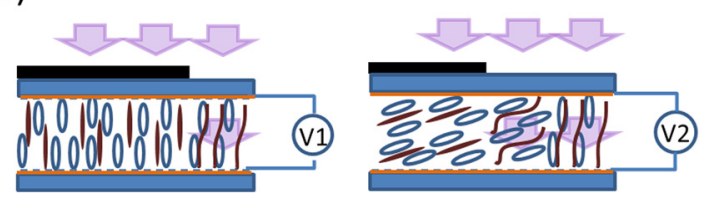

FIG. 2. (a) Schematic drawing of the fabrication setup; (b) dynamic control of the rectangular photomask and iris diaphragm; and (c), (d) UV exposure for generating spatial gradient refractive index $\left(V_{1}>V_{2}\right)$.

between two crossed polarizers with rubbing direction oriented at $45^{\circ}$ with respect to the polarizer. The color stripes result from the phase retardation of LC cell, indicating periodic variation in birefringence $\left(\mathrm{n}_{\mathrm{e}}-\mathrm{n}_{\mathrm{o}}\right)$. Adding a color filter $(\lambda=633 \mathrm{~nm})$ in the optical path, we can easily extract the optical path difference and the local birefringence of the sample cell based on the intensity variation. ${ }^{29}$

Figure 4 shows the diffraction pattern of this prism grating. A linearly polarized laser beam $(\lambda=633 \mathrm{~nm})$ with polarization parallel to the rubbing direction is normally incident on the sample cell. At $\mathrm{V}=0$, the strongest diffraction peak occurs at the -5 th order. As the voltage increases, it moves to the -3 rd order at $60 \mathrm{~V}$ and -2 nd order at $100 \mathrm{~V}$.
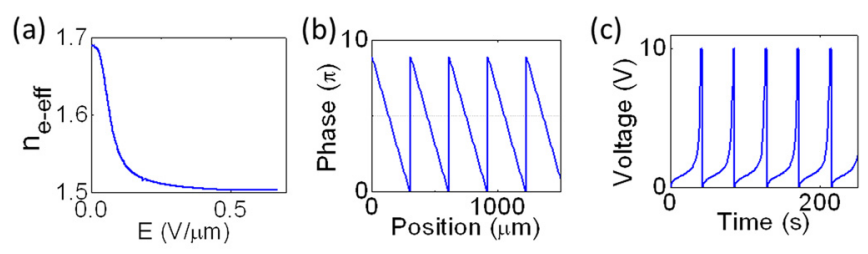

(d)

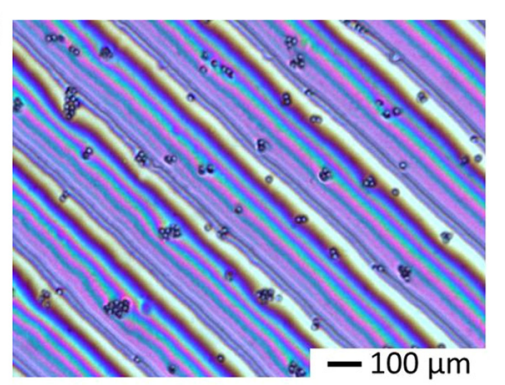

FIG. 3. (a) Electric field dependent $n_{e}$ of the LC precursor; (b) intended phase profile of micro-prism array (prism grating); (c) time dependent voltage chart for micro-prism array fabrication; and (d) polarized microscope image of a fabricated micro-prism array.

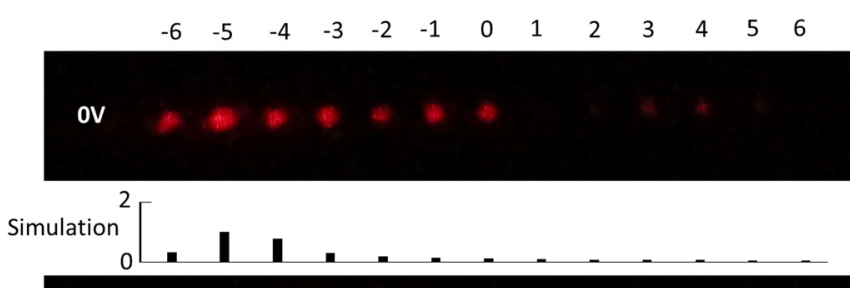

60V

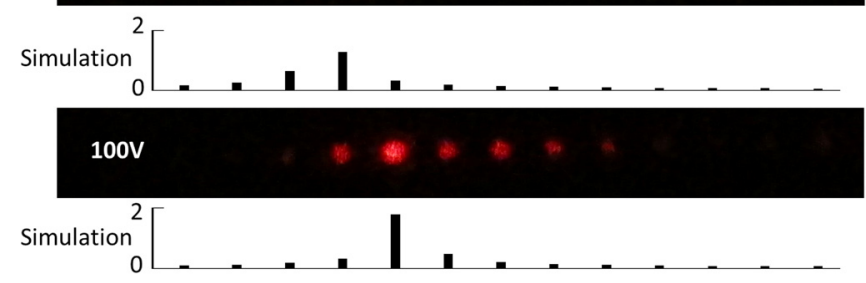

FIG. 4. Recorded diffraction patterns and the simulation results from Fourier transform (normalized to the -5 th order at $\mathrm{V}=0$ ).

This is because as the voltage increases, the LC directors are reoriented along the electric field direction so that the prism phase profile gradually becomes flatter. By analyzing the local birefringence, the phase contrast of the prism grating was measured to be $8.9 \pi$ at $0 \mathrm{~V}, 6.5 \pi$ at $60 \mathrm{~V}$, and $3.5 \pi$ at $100 \mathrm{~V}$. We simulate the diffraction pattern through the spatial Fourier transform of an ideal prism grating phase profile. Calculated values are normalized to the -5 th order at $0 \mathrm{~V}$. We found that the diffraction maximum moving towards the lower orders as voltage increases, showing the same trend as the experimentally observed diffraction patterns. The maximum diffraction order becomes more intense at $100 \mathrm{~V}$ than $60 \mathrm{~V}$ and $0 \mathrm{~V}$, in a good agreement with experimental results.

We also measured the switching time of this prism $\left(-5\right.$ th order) at $22^{\circ} \mathrm{C}$. Results are $870 \mu$ s for relaxation and $280 \mu$ s for rising (10\%-90\% change in transmission). The fast response time results from the small LC domain sizes of the polymer network. ${ }^{23}$ With this printing technique we are able to fabricate 1D (line shape) photonic devices easily, such as cylindrical micro-lens or LC phase gratings with any type of phase profile. However, this printing technique is not limited to 1D photonic devices only.

Next, we demonstrate another example for fabricating a circular (2D) adaptive LC lens. We use an iris diaphragm as photomask (Fig. 2(b)). With a motion controller, we are able to control the opening speed of the diaphragm. The accuracy of this setup can be further improved if we employ a high precision motorized iris diaphragm.

Figure 5(a) shows the optical image of a positive circular lens which has higher $n_{\text {e-eff }}$ at the center and lower $n_{\text {e-eff }}$ at the edges. The sample cell is placed on a white light table between two crossed polarizers. The color rings are caused by the birefringence gradient. To characterize the optical performance of this lens, we used an expanded He-Ne laser beam as the probing light source. The beam size was similar to the lens aperture, and the input polarization was parallel to the cell rubbing direction. A CCD camera was used to record the transmitted beam profile.

We measured the focal length of this LC lens at different voltages. Results are plotted in Fig. 5(b). Without LC lens, the laser beam was not focused. When the lens was placed in the 
(a)

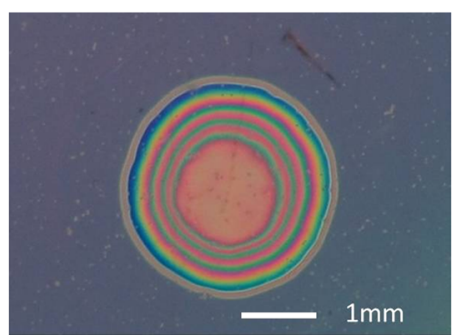

(c)

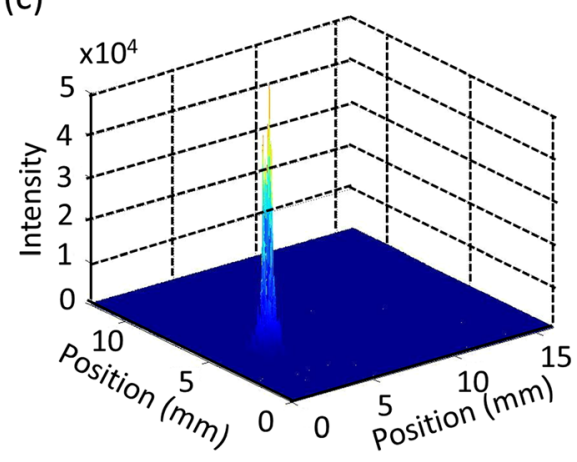

With Lens, OV (b)

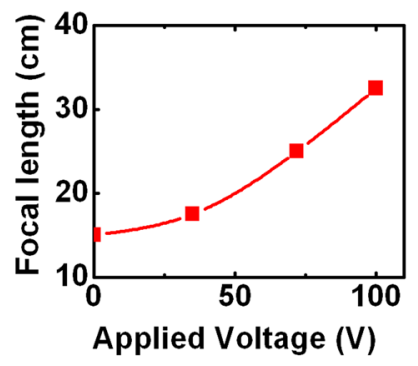

(d)

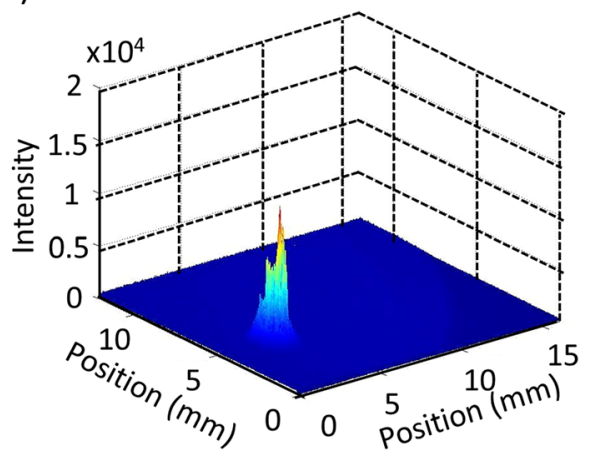

FIG. 5. (a) Optical image of a circular LC lens between crossed polarizers, (b) measured focal lengths at different voltages. (c) CCD images of the laser beam profile with lens, but $\mathrm{V}=0$, and (d) with lens at $\mathrm{V}=100 \mathrm{~V}_{\mathrm{rms}}$. light path, the outgoing laser beam was focused at the focal point of the positive lens. At $\mathrm{V}=0$, the focal length of this adaptive lens is $15 \mathrm{~cm}$. As the voltage increases from 0 to $100 \mathrm{~V}$, the measured focal length increases to $32 \mathrm{~cm}$. This is because when the voltage exceeds a threshold, the LC directors start to tilt up so the $n_{\text {e-eff }}$ difference between lens edge and lens center gets smaller. A smaller phase difference leads to a weaker optical power, i.e., longer focal length.

Various CCD images were taken at the focal point of the LC lens, as Figs. 5(c) and 5(d) depict. Figure 5(c) was taken after the expanded laser beam passing through the unpowered LC lens. An intense peak is observed because of the strong focusing effect of the lens. As the voltage increases, the focusing power gradually decreases. At $100 \mathrm{~V}$, the beam profile of the probing laser broadens, and the peak intensity decreases (Fig. 5(d)).

In conclusion, we developed a one-step printing technique for fabricating adaptive LC prism/grating/lens based on our scattering-free PNLC. For feasibility demonstration, we printed a microprism array with a periodicity of $\sim 300 \mu \mathrm{m}$ and a circular lens with a radius of $\sim 1.3 \mathrm{~mm}$. The switching time of these devices is in submillisecond range. In comparison with conventional microfabrication technique for adaptive LC photonic devices, our approach has following advantages: (1) Single step and short fabrication time; (2) easy to reconfigure; (3) low cost; and (4) submillisecond device response time. Although the resolution of this technique is not as high as photolithography, it offers a great flexibility for designing and fabricating the desired refractive index profile of an LC device. Since the device reconfiguration and fabrication are so simple, it will enable us to optimize the design efficiently based on the experimental data. These printed devices work for linearly polarized light only, which is similar to most adaptive LC devices.

The authors are indebted to the U.S. Air Force Office of Scientific Research (AFOSR) for financial support under
Contract No. FA95550-09-1-0170. H. Ren is grateful to the financial support of the National Research Foundation (NRF) of Korea, the Korea-China Joint Research Program under Grant No. 2012-0004814.

${ }^{1}$ R. L. Sutherland, V. P. Tondiglia, L. V. Natarajan, T. J. Bunning, and W. W. Adams, Appl. Phys. Lett. 64, 1074 (1994).

${ }^{2}$ P. F. McManamon, T. A. Dorschner, D. L. Corkum, L. Friedman, D. S. Hobbs, M. Holz, S. Liberman, H. Q. Nguyen, D. P. Resler, R. C. Sharp, and E. A. Watson, Proc. IEEE 84, 268 (1996).

${ }^{3}$ S. H. Kim and L. C. Chien, Opt. Express 12, 1238 (2004).

${ }^{4}$ J. Yan, Y. Li, and S. T. Wu, Opt. Lett. 36, 1404 (2011).

${ }^{5}$ W. Hu, A. Srivastava, F. Xu, J. T. Sun, X. W. Lin, H. Q. Cui, V. Chigrinov, and Y.-Q. Lu, Opt. Express 20, 5384 (2012).

${ }^{6}$ S. Sato, Jpn. J. Appl. Phys. 18, 1679 (1979).

${ }^{7}$ M. Ye and S. Sato, Jpn. J. Appl. Phys. 41, L571 (2002).

${ }^{8}$ Y. H. Fan, H. Ren, and S. T. Wu, Opt. Express 11, 3080 (2003).

${ }^{9}$ H. C. Lin and Y. H. Lin, Appl. Phys. Lett. 98, 083503 (2011).

${ }^{10} \mathrm{H}$. Ren and S. T. Wu, Introduction to Adaptive Lenses (Wiley, 2012).

${ }^{11}$ H. Ren, Y. H. Fan, and S. T. Wu, Appl. Phys. Lett. 82, 3168 (2003).

${ }^{12}$ S. Y. Huang, H. Y. Zheng, K. Y. Yu, B. Y. Huang, H. R. Lin, C. R. Lee, and C. T. Kuo, Opt. Mater. Express 2, 1791 (2012).

${ }^{13}$ H. Wu, W. Hu, H. C. Hu, X. W. Lin, G. Zhu, J. W. Choi, V. C. Chigrinov, and Y. Q. Lu, Opt. Express 20, 16684 (2012).

${ }^{14}$ S. T. Wu, Appl. Opt. 28, 48 (1989).

${ }^{15}$ H. Ren, D. W. Fox, B. Wu, and S. T. Wu, Opt. Express 15, 11328 (2007).

${ }^{16}$ S. Gauza, X. Zhu, S. T. Wu, W. Piecek, and R. Dabrowski, J. Disp. Technol. 3, 250 (2007).

${ }^{17}$ S. T. Wu and C. S. Wu, Appl. Phys. Lett. 53, 1794 (1988).

${ }^{18}$ Y. P. Huang, C. W. Chen, and Y. C. Huang, J. Disp. Technol. 8, 650 (2012).

${ }^{19}$ R. A. M. Hikmet, J. Appl. Phys. 68, 4406 (1990).

${ }^{20}$ R. A. M. Hikmet, Liq. Cryst. 9, 405 (1991).

${ }^{21}$ H. Ren, Y. H. Lin, and S. T. Wu, Opt. Commun. 261, 296 (2006).

${ }^{22}$ J. Sun, H. Xianyu, Y. Chen, and S. T. Wu, Appl. Phys. Lett. 99, 021106 (2011).

${ }^{23}$ J. Sun, Y. Chen, and S. T. Wu, Opt. Express 20, 20124 (2012).

${ }^{24}$ H. Ren, S. Xu, and S. T. Wu, Opt. Express 20, 26464 (2012).

${ }^{25}$ J. Sun, R. A. Ramsey, Y. Chen, and S. T. Wu, J. Disp. Technol. 8, 87 (2012).

${ }^{26}$ S. W. Kang, S. Sprunt, and L. C. Chien, Macromolecules 35, 9372 (2002).

${ }^{27}$ V. V. Sergan, T. A. Sergan, and P. J. Bos, Chem. Phys. Lett. 486, 123 (2010).

${ }^{28}$ I. C. Khoo and S. T. Wu, Optics and Nonlinear Optics of Liquid Crystals (World Scientific, Singapore, 1993).

${ }^{29}$ S. T. Wu, U. Efron, and L. D. Hess, Appl. Opt. 23, 3911 (1984). 